\title{
ORIGINAL
}

\section{DISTRIBUCIÓN Y TENDENCIA DE LOS FALLECIMIENTOS EN EL MEDIO HOSPITALARIO EN ESPAÑA DURANTE EL PERIODO 1997-2003}

\author{
Alberto Jiménez-Puente (1), Emilio Perea-Milla (2) y Francisco Rivas-Ruiz (2) \\ (1) Unidad de Evaluación. Hospital Costa del Sol, Marbella. \\ (2) Unidad de Investigación. Hospital Costa del Sol, Marbella. \\ CN 340, Km. 187. 29600 Marbella. Correo electrónico: ajpuente@ hcs.es \\ El Hospital Costa del Sol forma parte de la Red Yriss de Investigación en resultados de salud y servicios sanitarios.
}

\section{RESUMEN}

Fundamento: El lugar en que se produce la muerte de una persona depende de circunstancias de naturaleza demográfica, socioeconómica, cultural y asistencial El objetivo del estudio fue describir las variaciones en el porcentaje de muertes en hospitales entre las comunidades autónomas de España, diferenciando las que ocurrieron en urgencias y hospitalización, y explorar su relación con posibles variables explicativas.

Métodos: El estudio fue ecológico. Se estudió la tendencia del porcentaje de muertes en hospitales entre 1997 y 2003. Se calcularon los porcentajes de muertes en hospitales de cada comunidad autónoma en los años 2000-2002 a partir del Movimiento Natural de la Población y de la Encuesta de Establecimientos Sanitarios con Régimen de Internado que incluye información de todos los hospitales públicos y privados. Mediante regresión lineal simple se analizó su relación con variables demográficas, socioeconómicas, y asistenciales.

Resultados: El 53\% de los fallecimientos ocurrieron en hospitales (variando entre 37,3 y $68,4 \%$ en las distintas comunidades). El $10,7 \%$ de ellos en el área de urgencias (variando entre 6 y 14,5\%) y el resto en hospitalización.

Conclusiones: El porcentaje de muertes en hospitales fue mayor en las comunidades con menos población anciana y rural, más población extranjera y mayor frecuentación de urgencias. El porcentaje de muertes hospitalarias en urgencias fue mayor en las comunidades con peores indicadores socioeconómicos. La tendencia en los 7 años estudiados fue al aumento del porcentaje de muertes en hospitales.

Palabras clave: Mortalidad hospitalaria. Factores socioeconómicos.

Correspondencia:

Alberto Jiménez-Puente

Unidad de Evaluación. Hospital Costa del Sol

Marbella

Correo electrónico: ajpuente@hcs.es

\section{ABSTRACT \\ Distribution and Trend of Deaths within the Hospital Environment in Spain during the 1997-2003 period}

Background: Where a person's death occurs depends upon situations of a demographic, socioeconomic, cultural and healthcare-related nature. The objective of this study was that of describing the variations in the percentages of deaths in hospitals among the Autonomous Communities of Spain, distinguishing between those which occurred in emergency care and during hospitalization and delving into their relation with variables possibly providing an explanation thereto.

Methods: The study was an ecological one, the trend in the percentage of deaths in hospitals within the 1997-2003 period having been studied. The percentages of deaths in hospitals from each Autonomous Communities during the 2000-2002 period were calculated based on the Natural Population Movement and the Survey of InPatient Healthcare Establishments which includes information from all of the public and private hospitals. The relationship thereof to demographic, socioeconomic and healthcare-related variables was analyzed by single linear regression.

Results: A total of $53 \%$ of the deaths occurred in hospitals (ranging from $37.3 \%$ to $68.4 \%$ in the different Autonomous Communities). A total of $10.7 \%$ of the deaths occurred in emergency care (ranging from $6 \%$ to $14.5 \%$ ) and all others during hospitalization.

Conclusions: The percentage of deaths having occurred in hospitals was greater in the Autonomous Communities having a smaller elderly rural population, a larger foreign population and a higher degree of frequenting of emergency care. The percentage of hospital deaths in emergency care was greater in those Autonomous Communities having poorer socioeconomic indicators. The trend over the seven years studied was toward a rise in the percentage of deaths in hospitals.

Key words: Hospital mortality. Socioeconomic factors. 


\section{INTRODUCCIÓN}

El lugar en el que se produce la muerte de un paciente depende de circunstancias de naturaleza demográfica, socioeconómica, cultural y asistencial. En el caso de una patología aguda influye la accesibilidad a los servicios de urgencias, la agilidad de éstos para ingresar al paciente con vida y la efectividad de los tratamientos posteriores. Si la causa es una patología crónica diagnosticada y con pronóstico fatal, intervienen la decisión del paciente o sus familiares para acudir al hospital, la accesibilidad a una unidad de cuidados paliativos u otro servicio que ayude en la elección del lugar de la muerte y la práctica dispar de los hospitales para ingresar o dar de alta a los pacientes terminales.

En cuanto a las muertes acaecidas en los servicios de urgencias se ha descrito que la mayoría eran previsibles por la situación clínica de los pacientes a su llegada al hospital $^{1-3}$ y que, a menudo, habría sido más deseable que se hubieran producido en una planta del hospital o bien en el domicilio del paciente si su situación clínica era terminal $^{1,4}$.

$\mathrm{Ni}$ en la literatura médica ni en anuarios estadísticos hemos podido localizar referencias en España sobre la distribución del lugar de la muerte en población general. En otros países dicha información sí forma parte de las estadísticas oficiales. En 2002 el $43 \%$ de las muertes en Alemania ocurrieron en hospitales, en Estados Unidos el $49 \%$ y en Inglaterra y Gales el $67 \%$ 5-7. Respecto a la tendencia del proceso, en Estados Unidos se ha descrito un aumento progresivo de las muertes extrahospitalarias ${ }^{8-9}$. En Canadá se ha descrito la misma tendencia en el caso de pacientes con cáncer ${ }^{10}$ frente a la estabilidad que existe en el caso de Inglaterra ${ }^{11}$.

En cuanto a las variables que determinan el lugar de la muerte, en población general de otros países se ha descrito la influencia de variables socioeconómicas, de la oferta de camas en hospitales de agudos y de la disponibilidad de centros sociosanitarios ${ }^{8-9,12}$. La información al respecto es mucho mayor en el caso de los pacientes con cáncer ${ }^{13-17}$.

Por otra parte, estudios previos realizados en España han descrito importantes desigualdades socioeconómicas y en la oferta de servicios sanitarios entre las distintas comunidades autónomas (CCAA) $)^{18-19}$, así como la influencia de diversas características sociodemográficas sobre el riesgo de muerte, como el desempleo, el nivel educativo, el estado civil, el número de miembros del hogar, la actividad laboral o el apoyo social $^{18,20-24}$.

Nuestra hipótesis era que las muertes en hospitales serían más frecuentes en las CCAA con menos población anciana y rural, y que la proporción de muertes en urgencias se relacionaría directamente con la frecuentación hospitalaria e inversamente con las dotaciones de camas. En cuanto a la tendencia del proceso, la progresiva medicalización de la sociedad nos hacía esperar un aumento progresivo de las muertes en el hospital.

Los objetivos del estudio son: a) describir la proporción de fallecimientos en el medio hospitalario para el conjunto nacional y en las distintas CCAA, diferenciando las muertes en las áreas de urgencias y hospitalización; b) explorar la correlación ecológica del porcentaje de muertes en hospitales y del porcentaje de muertes hospitalarias en urgencias en las diferentes CCAA con variables de tipo demográfico, socioeconómico, asistencial y epidemiológico; c) describir la tendencia del porcentaje de muertes hospitalarias para el conjunto nacional en el periodo 1997 a 2003.

\section{MATERIAL Y MÉTODOS}

El estudio es de tipo analítico. Se obtuvo información de las muertes ocurridas en 
España y cada una de las CCAA entre 2000 y 2002 través del Movimiento Natural de la Población (MNP) elaborado por el Instituto Nacional de Estadística (INE) y de las producidas en hospitales a través de la Estadística de Establecimientos Sanitarios con Régimen de Internado (EESRI) elaborada por el Ministerio de Sanidad y Consumo. Para estudiar la tendencia de las muertes en hospitales se utilizaron datos globales a nivel nacional de 1997 a 2003.

La EESRI es una encuesta en la que participan obligatoriamente todos los hospitales públicos y privados, los cuales anualmente declaran su actividad asistencial y su dotación económica y de recursos humanos. Dentro del apartado de hospitalización, para grandes grupos de especialidades médicas, quirúrgicas, cuidados intensivos y unidades de larga estancia se recogen las altas por distintos motivos, incluidas las relativas a fallecimientos. Dentro del apartado de urgencias se recogen también los fallecimientos en este área. Los resultados de la EESRI se publican desagregados por CCAA.

La información se obtuvo a través de las páginas web de ambas instituciones ${ }^{25,26}$, en su totalidad en el caso del INE y completada con la solicitud directa de datos en el caso de la EESRI. Se calculó el porcentaje de fallecimientos hospitalarios respecto al total, excluyendo de éstos los correspondientes a españoles residentes fuera del territorio nacional, y el porcentaje de fallecimientos en urgencias respecto a los fallecimientos hospitalarios.

Se realizó un análisis de regresión lineal simple entre la proporción de muertes en el medio hospitalario de las 17 CCAA más la agrupación de Ceuta y Melilla y diversas variables de tipo: a) demográfico: porcentajes de población mayor de 65 años, población residente en medio rural (municipios de menos de 10.000 habitantes), población extranjera y hogares unipersonales (fuentes: explotación estadística del padrón municipal y censo); b) socioeconómico: tasas de analfabetismo en mayores de 10 años y de paro en mayores de 16 años, porcentaje de pensiones no contributivas y renta familiar disponible por habitante (euros anuales) (fuentes: censo, sistema de información de pensiones no contributivas del Ministerio de Trabajo y Asuntos Sociales y Sistema de Cuentas Regionales); c) asistencial: camas, urgencias e ingresos hospitalarios por 10.000 habitantes (fuentes: EESRI y censo) y d) causas de la muerte: porcentaje de muertes por cáncer y por enfermedades del aparato circulatorio (fuente: Movimiento Natural de la Población). Los indicadores demográficos y socioeconómicos de las distintas CCAA correspondieron al año 2001 y se accedió a ellos a través de las aplicaciones INEbase del INE y SIMA del Instituto de Estadística de Andalucía, accesibles a través de las páginas web de ambas instituciones ${ }^{27-28}$.

Dado que el número de unidades de estudio era pequeño, se utilizaron los coeficientes de correlación de Spearman. El nivel de significación se estableció en $p<0,05$. Se analizó, mediante la prueba de rachas, la tendencia de las series temporales de los porcentajes de muertes en hospitales y en urgencias. Los cálculos se realizaron con Lotus 123 y los análisis estadísticos con SPSS para Windows versión 9.

\section{RESULTADOS}

En los tres años a estudio se produjeron 1.081.414 fallecimientos en España, el 53\% de ellos en un centro hospitalario. En la tabla 1 se muestra la distribución por CCAA, en la que destaca Canarias con el 68,4\% de muertes en hospitales y Madrid y Cataluña con más del $60 \%$, y en el otro extremo Castilla La Mancha y Extremadura con sólo el $37,3 \%$ y el $40,8 \%$ de éxitus en hospitales, respectivamente.

El 5,7\% del total de las muertes ocurrieron en servicios de urgencias hospitalarios, des- 
Tabla 1

Distribución de fallecimientos en hospitales por Comunidad Autónoma, 2000-2002

\begin{tabular}{|c|c|c|c|c|c|}
\hline & Total & $\%$ en planta & $\begin{array}{l}\text { \% urgencias } \\
\text { hospitalarias }\end{array}$ & $\%$ hospitalarios & $\begin{array}{c}\% \text { fallecimientos } \\
\text { hopsitalarios } \\
\text { en urgencias* }\end{array}$ \\
\hline Andalucía & 182.067 & 44,3 & 6,1 & 50,4 & 12,1 \\
\hline Aragón & 38.302 & 47,1 & 3,1 & 50,1 & 6,1 \\
\hline Asturias & 36.743 & 44,8 & 4,5 & 49,3 & 9,1 \\
\hline Baleares & 21.444 & 54,1 & 4,1 & 58,2 & 7,1 \\
\hline Canarias & 35.821 & 59,2 & 9,2 & 68,4 & 13,5 \\
\hline Cantabria & 15.690 & 49,6 & 6,8 & 56,5 & 12,1 \\
\hline Castilla-La Mancha & 51.584 & 33,6 & 3,7 & 37,3 & 9,8 \\
\hline Castilla y León & 77.380 & 46,7 & 3,9 & 50,6 & 7,7 \\
\hline Cataluña & 170.227 & 53,1 & 7,3 & 60,4 & 12,1 \\
\hline Com. Valenciana & 111.845 & 42,7 & 5,3 & 48,0 & 11,0 \\
\hline Extremadura & 31.248 & 36,0 & 4,9 & 40,8 & 11,9 \\
\hline Galicia & 85.511 & 41,8 & 4,6 & 46,4 & 9,8 \\
\hline Madrid & 115.677 & 55,5 & 6,7 & 62,2 & 10,8 \\
\hline Murcia & 27.790 & 51,1 & 5,1 & 56,2 & 9,1 \\
\hline Navarra & 14.737 & 49,5 & 3,1 & 52,7 & 6,0 \\
\hline País Vasco & 54.957 & 49,6 & 6,5 & 56,1 & 11,5 \\
\hline La Rioja & 7.704 & 39,5 & 2,8 & 42,4 & 6,7 \\
\hline Ceuta y Melilla & 2.687 & 57,8 & 9,8 & 67,7 & 14,5 \\
\hline Total Nacional & 1.081 .414 & 47,4 & 5,7 & 53,0 & 10,7 \\
\hline
\end{tabular}

* Respecto al total de fallecimientos en hospitales (panta + urgencias).

tacando Ceuta y Melilla y Canarias con más del 9\%, frente a La Rioja, Aragón o Navarra en torno al 3\%. La proporción de los fallecimientos ocurridos en el área de urgencias respecto al total de éxitus en hospitales se situó en el 10,7\% a nivel nacional, oscilando entre el 14,5\% en Ceuta y Melilla o el 13,5\% en Canarias y cerca del 6\% en Aragón o Navarra.

En la tabla 2 se muestra el análisis de regresión lineal simple entre diversas variables socioeconómicas y asistenciales de las CCAA y las proporciones de muertes en el medio hospitalario y de muertes hospitalarias en urgencias.
El porcentaje de muertes en hospitales mostró asociaciones estadísticamente significativas con 3 variables demográficas y 1 asistencial, siendo mayor en las CCAA con menos población anciana, menos población rural y más población extranjera: coeficientes de regresión (B) -1,77, -0,37 y -1,16 y coeficientes de determinación $\left(\mathrm{R}^{2}\right)$ 0,45, 0,38 y 0,41 respectivamente, en todos los casos con $\mathrm{p}<0,01$, así como en aquellas comunidades con mayor frecuentación de urgencias: B 0,002, $\mathrm{R}^{2}$ 0,39, p 0,01.

El porcentaje de muertes hospitalarias en el área de urgencias mostró asociación estadísticamente significativa con 3 variables socioe- 
Tabla 2

Análisis de regresión lineal simple entre diversas características de las comunidades autónomas y los porcentajes de fallecimientos en hospitales y de fallecimientos hospitalarios en urgencias

\begin{tabular}{|c|c|c|c|c|c|c|}
\hline \multirow[b]{2}{*}{ Variables demográficas: } & \multicolumn{3}{|c|}{$\begin{array}{c}\text { Porcentaje de fallecimientos } \\
\text { en hospitales }\end{array}$} & \multicolumn{3}{|c|}{$\begin{array}{l}\text { Porcentaje de fallecimientos } \\
\text { hospitalarios en urgencias }+\end{array}$} \\
\hline & $\mathrm{B}$ & $\mathrm{R}^{2}$ & $\mathrm{p}$ & $\mathrm{B}$ & $\mathrm{R}^{2}$ & $\mathrm{p}$ \\
\hline$\%$ de población mayor de 65 años & $-1,77$ & 0,45 & 0,002 & $-0,41$ & 0,31 & 0,02 \\
\hline$\%$ de población rural & $-0,37$ & 0,38 & 0,009 & 0,05 & 0,06 & 0,36 \\
\hline$\%$ de población extranjera & 2,26 & 0,41 & 0,004 & $-0,04$ & 0,00 & 0,80 \\
\hline$\%$ de hogares unipersonales & $-1,16$ & 0,07 & 0,31 & $-0,66$ & 0,21 & 0,06 \\
\hline \multicolumn{7}{|l|}{ Variables socioeconómicas: } \\
\hline tasa de analfabetismo & 0,77 & 0,00 & 0,84 & 0,833 & 0,32 & 0,02 \\
\hline tasa de paro & $-0,48$ & 0,02 & 0,54 & 0,32 & 0,24 & 0,04 \\
\hline$\%$ de pensiones no contributivas & 0,55 & 0,02 & 0,62 & 0,22 & 0,41 & 0,004 \\
\hline renta familiar disponible por habitante & 0,002 & 0,16 & 0,10 & $-0,001$ & 0,12 & 0,16 \\
\hline \multicolumn{7}{|l|}{ Variables asistenciales: } \\
\hline camas por 10.000 habitantes & 0,71 & 0,05 & 0,37 & $-0,25$ & 0,12 & 0,16 \\
\hline urgencias por 10.000 habitantes & 0,002 & 0,39 & 0,01 & 0,0002 & 0,09 & 0,24 \\
\hline ingresos por 10.000 habitantes & 0,006 & 0,13 & 0,15 & 0,002 & 0,15 & 0,11 \\
\hline \multicolumn{7}{|l|}{ Causas de muerte } \\
\hline$\%$ de fallecimientos por cáncer & 0,54 & 0,02 & 0,62 & $-0,26$ & 0,05 & 0,37 \\
\hline$\%$ de fallecimientos por enf. circulatorias & $-1,20$ & 0,11 & 0,19 & 0,13 & 0,05 & 0,35 \\
\hline
\end{tabular}

* Respecto al total de fallecimientos en hospitales (panta + urgencias).

Tabla 3

Evolución de fallecimientos en hospitales, España 1997-2003

\begin{tabular}{|c|c|c|c|c|c|c|c|}
\hline & 1997 & 1998 & 1999 & 2000 & 2001 & 2002 & 2003 \\
\hline$\%$ fallecimientos en planta hospitalización & 43,8 & 44,5 & 45,4 & 47,0 & 47,3 & 47,7 & 48,0 \\
\hline$\%$ fallecimientos en urgencias hospitalarias & 6,5 & 6,4 & 6,2 & 5,8 & 5,6 & 5,7 & 6,0 \\
\hline$\%$ total fallecimientos en hospital & 50,3 & 50,9 & 51,6 & 52,7 & 52,9 & 53,5 & 54,0 \\
\hline$\%$ fallecimientos hospitalarios en urgencias & 12,9 & 12,5 & 12,0 & 11,0 & 10,5 & 10,7 & 11,1 \\
\hline
\end{tabular}

* Respecto al total de fallecimientos en hospitales (panta + urgencias).

conómicas y 1 demográfica, siendo mayor en las CCAA con mayor tasa de analfabetismo, paro y mayor porcentaje de pensiones no con- tributivas: (B $0,83,0,32$ y 0,22 y $\mathrm{R}^{2} 0,32,0,24$ y 0,41 respectivamente) y en aquéllas con menos población anciana (B -0,41 y $\mathrm{R}^{2} 0,31$ ). 
La EESRI ofrece algunos detalles más sobre las muertes ocurridas en hospitales: el $61 \%$ de las muertes en planta ocurrieron en especialidades médicas, el $14,6 \%$ en cuidados intensivos, el 13,1\% en servicios quirúrgicos y el 7,8\% en unidades de larga estancia.

En la tabla 3 se muestra la evolución de las muertes en hospitales entre 1997 y 2003 . Se aprecia un aumento del porcentaje de fallecimientos en hospitales de aproximadamente un $0,5 \%$ anual en el conjunto del país, desde el 50,3\% en 1997 al 54,0\% en 2003 (test de rachas: $\mathrm{p} 0,10$ ), así como una tendencia prácticamente estable de los fallecimientos hospitalarios en el área de urgencias, desde el $12,9 \%$ en 1997 al $11,1 \%$ en 2003.

\section{DISCUSIÓN}

En nuestro estudio, el porcentaje de muertes en hospitales de las distintas CCAA España mostró una buena correlación con las variables demográficas analizadas, mientras que el porcentaje de muertes hospitalarias en urgencias lo hizo con las variables socioeconómicas.

En el conjunto de España, el 53\% de las muertes ocurrieron en hospitales. En comparación con otros países, dicho porcentaje se sitúa en un nivel intermedio entre la situación de Alemania y EEUU (43 y 49\% respectivamente) y la de Inglaterra y Gales $(67 \%)^{5-7}$.

El segundo hallazgo destacable del estudio fue la amplia variabilidad en el lugar de la muerte entre CCAA. En la literatura ya se había comunicado dicha variedad entre distintos países en pacientes con patologías similares, lo que se ha considerado reflejo de la gran medida en que está influida por factores socioculturales o asistenciales ${ }^{9-12,15}$.

En nuestro estudio, los menores porcentajes de muertes en hospitales se dieron en las dos CCAA con más población rural: Casti-
lla-La Mancha y Extremadura, ambas con un porcentaje de población residente en municipios de menos de 10.000 habitantes superior al $50 \%$ en $2002^{27}$. El carácter rural de una comunidad se podría relacionar con una mentalidad y estilos de vida más tradicionales que lleven a asumir con más normalidad la muerte en el propio hogar ${ }^{14} \mathrm{o}$ con una peor accesibilidad física o geográfica a los servicios sanitarios.

Estimamos que el acceso al sistema sanitario, prácticamente universal en España, no está condicionado de forma relevante por factores económicos, aunque siempre es posible una relación entre estos y comportamientos sociales, como la decisión de acudir al hospital.

En estudios previos realizados en otros países se ha descrito en población general que la muerte en hospitales de agudos es más frecuente en pacientes afroamericanos ${ }^{8} \mathrm{y}$ que se correlaciona positivamente con la oferta de camas ${ }^{12}$ y negativamente con la disponibilidad de centros sociosanitarios ${ }^{9}$. En Estados Unidos se ha comunicado que el lugar de la muerte está más influido por la decisión de los pacientes al acudir al hospital que por la práctica de dar de alta a los pacientes terminales ${ }^{9}$.

La mayoría de los estudios sobre las variables relacionadas con el lugar de la muerte se han centrado en el caso de pacientes con cáncer. El porcentaje de los que fallecen en un hospital oscila entre el 26-35\% en el Reino Unido $^{11,15}, 52 \%$ en Estados Unidos ${ }^{13}, 59 \%$ en España ${ }^{14}$ y $74 \%$ en Canadá ${ }^{10}$. Se ha observado que la probabilidad de morir en un hospital es mayor en pacientes afroamericanos $^{13}$, de menor nivel socioeconómico ${ }^{15-17}$, cuanto mayor es la lejanía geográfica al hospital o la carencia de centros sociosanita$\operatorname{rios}^{15}$. En España se ha descrito que la muerte en el hogar de los pacientes con cáncer era más frecuente entre los residentes en el medio rural y en aquellos con mayor nivel socioeconómico ${ }^{14}$. En la literatura también 
se ha descrito el contraste entre la preferencia por la muerte en el propio hogar de la mayor parte de los pacientes con cáncer y la realidad de una mayoría de muertes hospitalarias $^{9,16}$.

En cuanto a la tendencia del proceso, los datos de los años disponibles mostraron una evolución ascendente de las muertes hospitalarias en España, contraria a la descrita en otros países ${ }^{8-10}$.

Respecto al 5,7\% de muertes en servicios de urgencias hospitalarios se sitúan por debajo del 7-8\% comunicados en EEUU ${ }^{6,8}$. Dicho porcentaje mostró una correlación estadísticamente significativa con 3 de las 4 variables socioeconómicas estudiadas, en todos los casos en el sentido de ser más frecuente en las CCAA con peores indicadores socioeconómicos. Es conocida la relación entre desigualdades sociales, estado de salud, oferta y acceso a los servicios sanita$\operatorname{rios}^{18-19}$. El mecanismo por el que las CCAA más desfavorecidas presentan mayor porcentaje de muertes en urgencias podría ser la sobrecarga asistencial de estos servicios en comunidades más pobres y, por tanto, con mayor carga de enfermedad.

En el informe sobre desigualdades sociales en salud en España ${ }^{18}$, se clasificaron las CCAA según un índice sintético de privación material basado en las tasas de desempleo y analfabetismo. Las comunidades con situación más negativa de dicho índice, como Ceuta-Melilla, Canarias, Andalucía o Extremadura, mostraron en nuestro estudio los porcentajes más altos de muertes hospitalarias en urgencias. Por el contrario, las CCAA mejor situadas según aquel índice: La Rioja, Aragón o Navarra, presentaron los menores porcentajes de muertes hospitalarias en urgencias según nuestros datos.

Si se puede hablar de las mejores circunstancias para la muerte de un paciente terminal, probablemente sean en su domicilio, en un ambiente tranquilo y rodeado de sus seres queridos. En el otro extremo, los servicios de urgencias suelen ser un lugar estresante y en el que habitualmente se limita el acompañamiento de familiares. En diversos estudios realizados en España se ha comunicado que la muerte era esperable en gran parte de los fallecidos en urgencias ${ }^{1-3}$ y que la mayoría de ellos murieron solos ${ }^{3}$. Consideramos deseable, tanto desde un punto de vista médico como humano, que el porcentaje de fallecimientos en urgencias, que en la actualidad ronda el $6 \%$ del total y el $11 \%$ de los ocurridos en hospitales, siga reduciéndose en lo posible.

La amplia variedad en la proporción de muertes en urgencias en las distintas regiones esconde, sin duda, variaciones mucho mayores entre hospitales. Las muertes en urgencias quedan fuera de las evaluaciones de la mortalidad intrahospitalaria en términos de calidad asistencial de sistemas implantados recientemente en España y hace más años en otros países ${ }^{29-30}$. Nuestra propuesta al respecto es que los conjuntos mínimos básicos de datos (CMBD) deberían incorporar todas las muertes ocurridas en hospitales, ya sucedan en hospitalización o en urgencias ${ }^{31}$.

Como limitaciones del estudio, las defunciones recogidas en el MNP se asignan a la provincia de residencia del fallecido, mientras que los hospitales declaran en la EESRI todas las muertes ocurridas en cada centro, independientemente de la residencia del paciente, por tanto los flujos de pacientes de unas CCAA a otras podrían suponer un sesgo si correspondieran a patologías con elevada mortalidad intrahospitalaria. Estimamos que la exhaustividad de las dos fuentes de información utilizadas: MNP y EESRI es muy alta. La primera procede del proceso legal y obligatorio de la certificación de la muerte. Por otra parte, son bien conocidas las limitaciones de los estudios ecológicos derivadas de su metodología ${ }^{32}$, entre ellas la denominada falacia ecológica. Por ello, no es posible garantizar que las relaciones entre 
variables observadas en nuestro estudio se reproduzcan en términos de causalidad a nivel individual.

El lugar en que se produce la muerte tiene importantes implicaciones sobre la organización de los servicios sanitarios, pues según cómo se distribuya en cada región, hará necesario reforzar los servicios de urgencias, de hospitalización, los cuidados domiciliarios o en centros sociosanitarios. Se requieren estudios longitudinales y basados en datos individuales, para aclarar en qué medida el lugar de la muerte está influido por la naturaleza de las patologías causantes, por las preferencias de los pacientes o sus familiares o por la práctica de los profesionales sanitarios.

\section{AGRADECIMIENTOS}

Al Instituto de Información Sanitaria del Ministerio de Sanidad y Consumo, que aportó los datos solicitados de la EESRI.

\section{BIBLIOGRAFÍA}

1. Tudela P, Módol JM. Urgencias hospitalarias. Med Clin (Barc) 2003;120:711-6.

2. Miró O, de Dios A, Antonio MT, Sánchez M, Borrás A, Millá J. Estudio de la mortalidad en un servicio de urgencias de medicina hospitalario: incidencia, causas y consecuencia. Med Clin (Barc) 1999;112:690-2.

3. Maroto O, Llorente A, Casanueva M, Álvarez B, de la Riva G. ¿Son los servicios de urgencias un lugar adecuado para morir? An Med Interna (Madrid) 2004;21:585-9.

4. Miró O, Sánchez M, Coll-Vinent B, Millá J. Indicadores de calidad en urgencias: comportamiento en relación con la presión asistencial. Med Clin (Barc) 2001; 116:92-7.

5. Statistisches Bundesamt Deutschland.Health status and reasons for treatment [consultado 9-1-2006]. Disponible en http://www.destatis.de/themen/e/ thm_bevoelk.htm
6. National Center for Health Statistics. Deaths from 39 selected causes by place of death, status of decedent when death occurred in hospital or medical center, and age: United States, 1999-2002. [consultado 9-1-2006]. Disponible en: http://www.cdc. gov/nchs/data/dvs/MortFinal2002_WorkTable307.pdf

7. Office for National Statistics. Mortality statistics. Review of the Registrar General of deaths in England and Wales, 2002. [consultado 9-1-2006]. Disponible en: http://www.statistics.gov.uk/downloads/theme_health/DH1_35_2002/DH1no35.pdf

8. Flory J, Yinong YX, Gurol I, Levinsky N, Ash A, Emanuel E. Place of death: US trends since 1980. Health Aff (Millwood) 2004; 23:194-200.

9. Tolle SW, Rosenfeld AG, Tilden VP, Park Y. Oregon's low in-hospital death rates: what determines where people die and satisfaction with decisions on place of death? Ann Intern Med 1999; 130:681-5.

10. Burge F, Lawson B, Johnston G. Trends in the place of death of cancer patients, 1992-1997. CMAJ $2003 ; 168: 265-70$.

11. Higginson IJ, Astin P, Dolan S. Where do cancer patients die? Ten-year trends in the place of death of cancer patients in England? Palliat Med 1998; 12:353-63.

12. Pritchard RS, Fisher ES, Teno JM, Sharp SM, Reding DJ, Knaus WA et al. Influence of patients preferences and local health system characteristics on the place of the death. J Am Geriatr Soc 1998; 46:1320-1.

13. Bruera E, Russell N, Sweeney C, Fisch M, Palmer L. Place of death and its predictors for local patients registered at a comprehensive cancer center. J Clin Oncol 2002;20:2127-33.

14. Catalán JG, Pons O, Recober A, Avella A, Carbonero JM, Benito E et al. Dying of cancer. The place of death and family circumstances. Med Care 1991; 29:841-52.

15. Gatrell AC, Harman JC, Francis BJ, Thomas C, Morris SM, McIllmurray M. Place of death: analysis of cancer deaths in part of North West England. J Publ Hlth Med 2003; 25:53-8.

16. Tang ST, McCorkle R. Determinants of place of death for terminal cancer patients. Cancer Invest 2001;19:165-80.

17. Grundy E, Mayer D, Young H, Sloggett A. Living arrangements and place of death of older people 
with cancer in England and Wales: a record linkage study. Br J Cancer 2004;91:907-12.

18. Navarro V, Benach J. Desigualdades sociales en España. Informe de la Comisión Científica de estudios de las desigualdades sociales de Salud en España. Rev Esp Salud Pública 1996;70:505-636.

19. González B, Urbanos RM, Ortega P. Oferta pública y privada de servicios sanitarios por comunidades autónomas. Gac Sanit 2004;18(Supl.1):82-9.

20. Regidor E, Calle ME, Domínguez V, Navarro P. Mortalidad según características sociales y económicas: Estudio de Mortalidad de la Comunidad Autónoma de Madrid. Med Clin (Barc) 2001;116:726-31.

21. Domínguez MF, Borrell C. Mortalidad y privación socioeconómica en las secciones censales y distritos de Barcelona. Gac Sanit 2005;19:363-9.

22. Nebot M, Lafuente JM, Tomás Z, Borrell C, Ferrando J. Efecto protector del apoyo social en la mortalidad en población anciana: en estudio longitudinal. Rev Esp Salud Pública 2005;76:673-82.

23. Benach J, Yasui Y, Borrell C, Sáez M, Pasarin MI. Material deprivation and leading causes of death by gender: evidence from a nationwide small area study. J Epidemiol Community Health 2001; 55:239-45.

24. Benach J, Yasui Y, Martínez JM, Borrell C, Pasarin MI, Daponte A. The geography of the highest mortality areas in Spain: a striking cluster in the south- western region of the country. Occup Environ Med 2004;61:280-1.

25. Instituto Nacional de Estadística. Movimiento Natural de la Población. [consultado 9-1-2006]. Disponible en: http://www.ine.es/inebase/index. $\mathrm{html}$

26. Ministerio de Sanidad y Consumo. Encuesta de Establecimientos Sanitarios con régimen de internado. [consultado 9-1-2006]. Disponible en: http://www.msc.es/Diseno/sns/sns_sistemas_informacion.htm

27. Instituto Nacional de Estadística. Explotación estadística del padrón. [consultado 9-02-2006]. Disponible en: http://www.ine.es/inebase/index.html

28. Instituto de Estadística de Andalucía. Sistema de Información Multiterritorial de Andalucía. [consultado 9-1-2006]. Disponible en: http://www.juntadeandalucia.es/institutodeestadistica/sima/index $2 . h t m$

29. Iasist. Hospitales Top-20. Benchmarks para la excelencia. [consultado 9-1-2006]. Disponible en: http://www.iasist.com/iasist.html

30. Jacobson B, Mindell J, McKee M. Hospital mortality league tables. Questions what they tell you and how useful they are. BMJ 2003;326:777-8.

31. Jiménez A, López J, Lara A. La frontera entre urgencias y hospitalización en el análisis de la mortalidad hospitalaria. Rev Clin Esp 1999; 199: 813-6.

32. Morgenstern H. Ecologic studies in epidemiology. Annu Rev Public Health 1995; 16:61-81. 